\title{
Evaluating Edge Detection through Boundary Detection
}

\author{
Song Wang, Feng Ge, and Tiecheng Liu \\ Department of Computer Science and Engineering, University of South Carolina, Columbia, SC 29208, USA
}

Received 27 February 2005; Revised 6 June 2005; Accepted 30 June 2005

\begin{abstract}
Edge detection has been widely used in computer vision and image processing. However, the performance evaluation of the edgedetection results is still a challenging problem. A major dilemma in edge-detection evaluation is the difficulty to balance the objectivity and generality: a general-purpose edge-detection evaluation independent of specific applications is usually not well defined, while an evaluation on a specific application has weak generality. Aiming at addressing this dilemma, this paper presents new evaluation methodology and a framework in which edge detection is evaluated through boundary detection, that is, the likelihood of retrieving the full object boundaries from this edge-detection output. Such a likelihood, we believe, reflects the performance of edge detection in many applications since boundary detection is the direct and natural goal of edge detection. In this framework, we use the newly developed ratio-contour algorithm to group the detected edges into closed boundaries. We also collect a large data set (1030) of real images with unambiguous ground-truth boundaries for evaluation. Five edge detectors (Sobel, LoG, Canny, Rothwell, and Edison) are evaluated in this paper and we find that the current edge-detection performance still has scope for improvement by choosing appropriate detectors and detector parameters.
\end{abstract}

Copyright () 2006 Hindawi Publishing Corporation. All rights reserved.

\section{INTRODUCTION}

Edge detection is a very important feature-extraction method that has been widely used in many computer vision and image processing applications. The basic idea of most available edge detectors is to locate some local object-boundary information in an image by thresholding and skeletonizing the pixel-intensity variation map. Since the earliest work by Julez [1] in 1959, a huge number of edge detectors has been developed from different perspectives (e.g., [2-9]). A very natural and important question is then: which edge detector and detector-parameter settings can produce better edgedetection results? This strongly motivates the development of a general and systematic way of evaluating the edge-detection results.

Prior edge-detection evaluation methods can be categorized in several ways. First, they can be classified as subjective and objective methods. The former uses the humanvisual observation and decision to evaluate the performance of edge detection. Given the inherent inconsistency in human perception, subjective evaluation results may exhibit a large variance for different observers. In objective methods, quantitative measures are defined based solely on images and the edge-detection results. Second, edge-detection evaluation methods can be categorized according to their requirement of the ground truth. With the ground truth, edge detection can be quantitatively evaluated in a more credible way. Without the ground truth, some local coherence information [10] is usually used to measure the performance. Third, edge-detection evaluation methods can be categorized based on test images: synthetic-image-based methods and real-image-based methods. A more detailed discussion on various edge detectors and edge-detection evaluation methods can be found in [11].

Although many edge-detection evaluation methods have been developed in the past years (e.g., [11-15]), this is still a challenging and unsolved problem. The major challenge comes from the difficulty in choosing an appropriate performance measure of the edge-detection results. In most applications, edge detection is used as a preprocessing step to extract some low-level boundary features, which are then fed into further processing steps, such as object finding and recognition. Therefore, the performance of edge detection is difficult to define without embedding it into certain applications. However, if edge detection is evaluated based on the performance of a special application $[13,16]$, such an evaluation may not be applicable to other applications. This introduces a well-known dilemma inherent in the edge-detection evaluation: general-purpose evaluation is difficult to define, while evaluation based on a specific application reduces the generality of the evaluation method.

To resolve the dilemma and considering the different categories of prior methods, we propose four desirable features for a good edge-detection evaluation method. 
(1) Generality: the evaluation measure should be well quantified yet generally applicable.

(2) Objective evaluation with ground truth: the evaluation measure should be objective to avoid potential inconsistency in subjective evaluation. It should also use ground truth to achieve a credible evaluation.

(3) Real image: real (noisy) images should be used for evaluation, as prior research has revealed that conclusions drawn from synthetic images are usually not applicable to real images.

(4) Large data set: a convincing edge-detection evaluation should be conducted in a large set of real images and the results should be drawn through statistical analysis.

Considering these desirable features, we present in this paper a new method for edge-detection evaluation. The major novelty of this method is to evaluate edge detection in the framework of boundary detection, that is, detecting a full closed boundary of the salient object in an image. The basic idea is straightforward: although edge-detection results have been used for different applications, one of the fundamental goals of edge detection in many applications is to detect some compact object-boundary information that can facilitate further image processing. As shown in Figure 1, from the detected edges, we can estimate how likely it is that the complete geometry of the salient object boundaries present in this image will be determined. The likelihood of determining the full object boundaries, to some extent, reflects the edge-detection performance on many applications, such as object recognition, tracking, and image retrieval, although those applications may not explicitly have a component to derive full object boundaries out of the edge-detection results. Therefore, using this boundary-detection likelihood to evaluate edge detection not only makes the problem well defined but also avoids overly sacrificing of the generality of the evaluation.

To achieve this goal, we collect a set of real images each of which consists of an unambiguous foreground salient object and a noisy background. In these images, the groundtruth object boundary can be unambiguously extracted by manual processing, which enables an objective and quantitative measurement of the boundary-detection performance. A major component in our framework is to find a reliable algorithm for detecting a salient closed boundary from the edge-detection results. In this paper, we use our recently developed ratio-contour algorithm to achieve this goal. In [17], we show the superiority of the ratio-contour algorithm over other existing algorithms for detecting salient closed boundary in a set of detected edges. In particular, this ratio-contour algorithm integrates the Gestalt laws of closure, continuity, and proximity, which are well-known properties to describe the perceptual saliency of an object boundary. In addition, it guarantees global optimality in boundary detection without requiring any kinds of initialization.

A related but simpler study was carried out by Baker and Nayar [12], where edge detection was evaluated using some specified global coherence measures. In particular, they constructed a set of images in which the ground-truth boundaries were known to be a single straight line, two

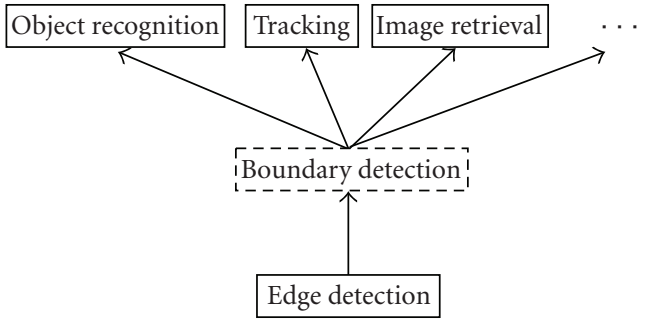

Figure 1: The likelihood of extracting full boundaries from detected edges reflects the performance of edge-detection algorithms in general applications.

parallel lines, two intersected lines, or an ellipse. In this way, the edge detection could be evaluated by checking these edges' conformance to the four a priori known global coherence measures. In this paper, the likelihood of locating a foreground object boundary can be treated as a more general global coherence measure, which is applicable to much wider classes of real images than the specified measures used in [12]. Furthermore, without constructing and applying the ground truth, Baker and Nayar's method [12] requires that the test image contains no or very weak background noise. This substantially reduces its applicability and generality because good edge detections should not only extract more salient-boundary features, but also suppress the background noise. The evaluation method proposed in this paper addresses the problem effectively by testing algorithms on real noisy images and incorporating the ground-truth boundaries.

In the remainder of this paper, Section 2 gives a precise formulation of evaluating edge detection in terms of boundary detection. Section 3 discusses the detailed settings for each component of our edge-detection evaluation. Section 4 briefly describes the edge detectors used for evaluation in this paper. For this paper, we chose five edge detectors: Sobel [9], LoG [18], Canny [3], Rothwell [19], and Edison [20] for evaluation. Section 5 reports and analyzes the evaluation results on the collection of 1030 real images. A short conclusion, together with a brief discussion on future work, is given in Section 6.

\section{EVALUATION FRAMEWORK}

As mentioned above, our goal is to evaluate edge detection according to the likelihood of locating the ground-truth object boundary from edge-detection results. Therefore, we need first to have a boundary-detection algorithm that can locate a salient closed boundary from a set of detected edges. The coincidence between the detected boundary and the ground-truth object boundary is then used to measure the performance of the edge detection, as shown in Figure 2. Following many prior human-vision and computer-vision studies, we formulate the boundary detection as a boundarygrouping process, in which a closed boundary is obtained by identifying a subset of the detected edges and then connecting them sequentially into a closed boundary. 


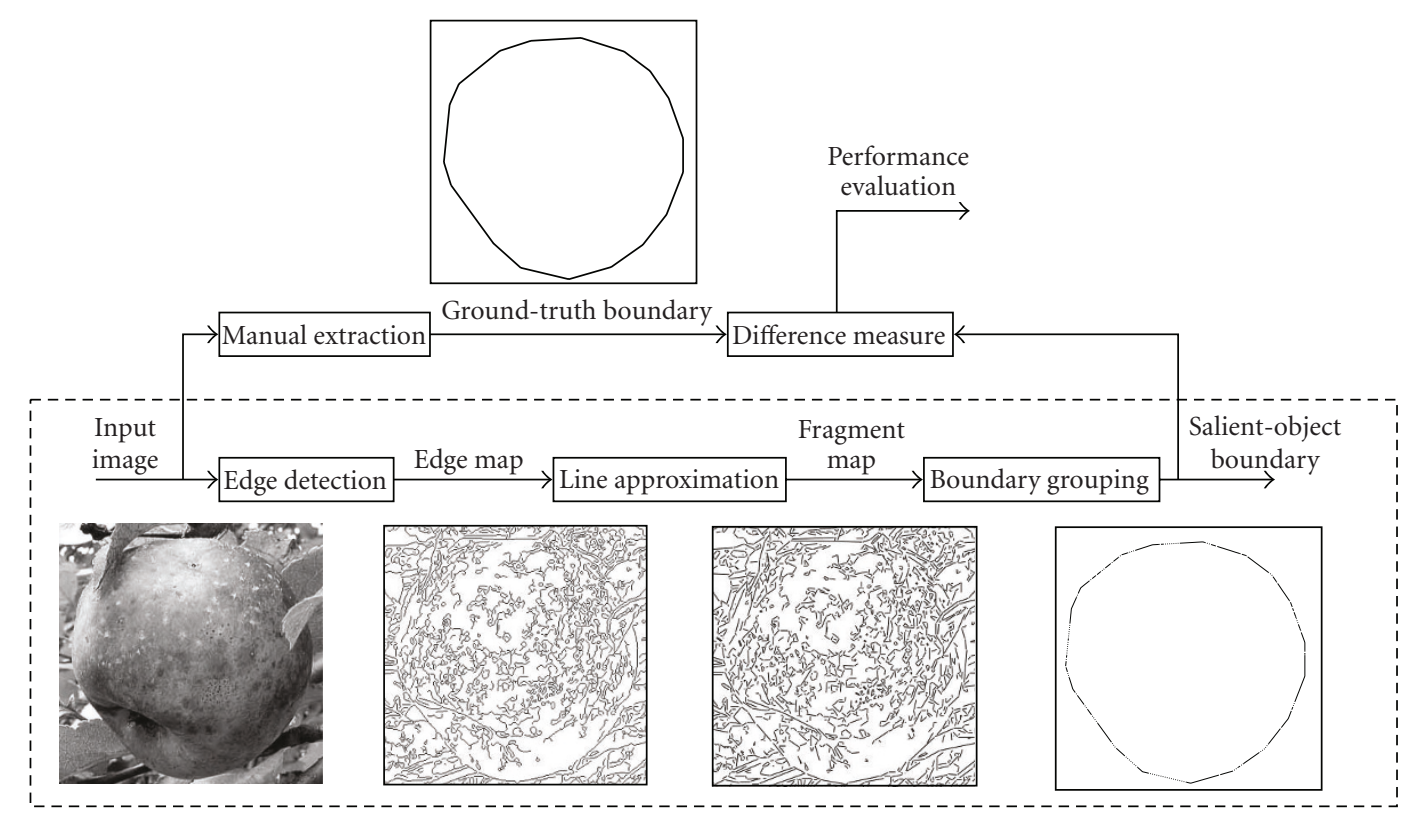

Figure 2: An illustration of the framework for evaluating edge detection through boundary detection. The three components in the dashedcurve box comprise a boundary detection system.

Starting from a real image, this boundary-detection system consists of three sequential components: edge detection, line approximation, and boundary grouping, as shown in the dashed-line box in Figure 2.

In the first component, an edge detector, together with some specified detector parameters, is used to detect a set of edges, that is, sequences of connected edge pixels, from an input image. These edge pixels may result from the salient object boundary or background noise. Note that an 8-connected pixel neighborhood system is usually used to trace the connected edge pixels. In the second component, our goal is to derive the edge direction, that is, the boundary direction at each detected edge pixel, which plays an important role in measuring the boundary saliency and guiding the boundary grouping. The edges augmented with direction information are called fragments in this paper. In the last component, our goal is to identify a subset of the fragments and sequentially connect them into a closed boundary that is to be aligned with the most salient object in the input image. To achieve the boundary closure, we need to fill in the gaps between the neighboring fragments in this boundary connection.

There are several important problems that need to be addressed in this framework to make this evaluation more convincing. First, it is particularly important to collect a large set of test images that are suitable for evaluation. On the one hand, the collected images should be real images with certain variety and complexity. For example, they should contain various types and levels of noise. On the other hand, the ground-truth object boundary must be able to be manually extracted in an unambiguous way, that is, from the same image, different people should perceive the same salient object. This problem will be discussed in detail in Section 3.1.
Second, we need to choose a set of typical edge detectors and their typical detector parameters for evaluation. As the first component in this framework, different edge detectors or different detector parameters produce different edge maps on the same image. We then compare and evaluate these edge maps by comparing the accuracy of boundary detection in terms of the ground-truth boundary in this image. One consideration is that our selected edge detectors should cover both classical and recent ones. The selection of edge detectors and their parameters will be discussed in Section 4.

Third, we need to choose an appropriate algorithm to estimate and represent fragments, that is, edges with direction information. Some edge detectors [3] have a nonmaximum suppression step, which provides an estimation of the edge directions. However, the nonmaximum suppression step usually only considers a small neighborhood and the estimated directions are very sensitive to the image noise, as explained in detail in $[19,20]$. To address this problem and also make this edge-direction estimation component consistent in all edge detectors, we adopt a line-approximation algorithm to fit the edges by some line segments, providing more accurate and robust edge direction estimation. This way, each fragment is in the form of a straight line segment, as shown in Figure 2. We will discuss this in detail in Section 3.2.

Fourth, we need to find a boundary-grouping algorithm that aims at detecting the salient closed boundary from the fragments. Since the ground-truth boundary is constructed by manual processing, the boundary-grouping algorithm should detect the salient object boundaries that are consistent with the human vision system. In this paper, we use the ratio-contour algorithm for boundary grouping, which will be discussed in Section 3.3. 
Finally, we need to choose a quantitative criterion for measuring the coincidence between the detected boundary and the ground-truth boundary. Such a criterion should be insensitive to possible small errors introduced in the manual construction of the ground truth. We will discuss this problem in Section 3.4.

\section{EVALUATION SETTINGS}

\subsection{Test-image database}

We collected 1030 real natural images from the internet, digital photos, and some well-known image databases such as Corel for the proposed edge-detection evaluation. We carefully examined each image before including it into our testimage database. A particular requirement is that each image contains a single perceptually unambiguous foreground salient object and a noisy background. Figure 3 demonstrates several sample images in our test-image database. In these images, we extract the ground-truth object boundary by simple manual processing. Note that, with the perceptual unambiguity in distinguishing the foreground object and background noise, we can assume that the groundtruth boundary is unique for each image and is largely independent on the specific person who manually extract this ground-truth boundary. Samples of the extracted groundtruth boundaries are also shown in Figure 3. We intentionally collect images with various foreground objects, such as human, animal, vehicle, building, and so forth. To facilitate the evaluation, all the images are unified to 256-bit gray-scale images in PGM format, with a size in the range of $80 \times 80$ to $200 \times 200$.

Note that our real-image database has completely different use to the real-image database in the Berkeley benchmark [21], where the goal is to evaluate various region-based image-segmentation algorithms. In the Berkeley benchmark, an image may contain many complex structures and therefore, the manual segmentation of the same image may be quite different across different people. If we use the Berkeley benchmark for our edge-detection evaluation, it would pose a much higher requirement for the boundary-grouping algorithm and greatly complicate the measure-criteria definition given that there is no unique ground truth. On the contrary, our carefully selected images have no such problems: the ground truth has no ambiguity and detecting a single salient boundary from the noisy background makes fewer demands on the boundary-grouping algorithm. We also believe that our collected images are sufficient, to a large extent, for the edge-detection evaluation because, in essence, edge detection is a local processing involving with the foreground structure and background noise, both of which have been included in all our collected images. However, our image database may not be suitable for general image-segmentation evaluation, because many natural images contain hierarchical structures that are not present in our collected images.

Our image database also differs from the real-image database used in the South Florida benchmark [11], where the goal is also for edge-detection evaluation. Because the South Florida benchmark evaluates edge detection using
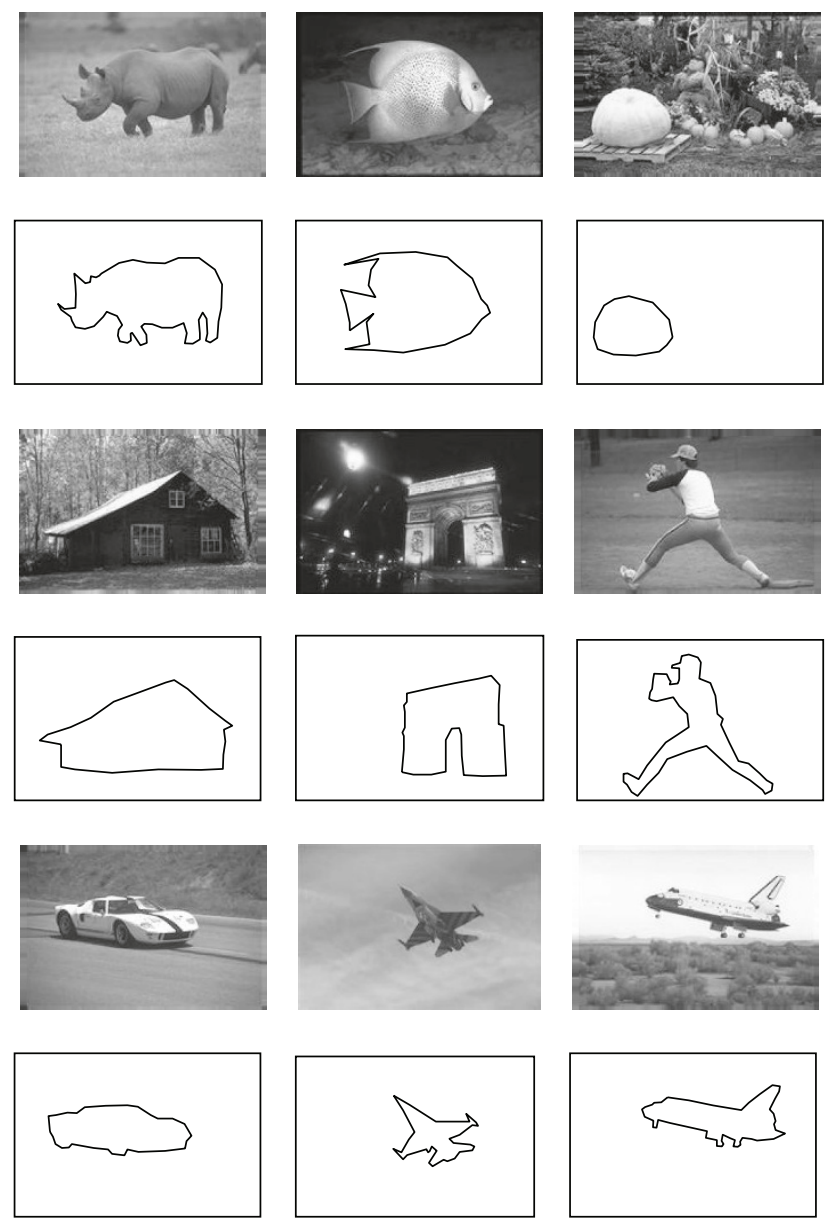

FIgURE 3: Nine sample images in our image database and the ground-truth boundaries manually extracted from them.

a subjective method, the images used in its benchmark contain more complicated structures and there exists no single ground-truth boundary. As our main focus is edge-detection evaluation instead of studying human psycho-visual differences in image understanding, we only select test images with unambiguous foreground and background, which in fact makes objective and quantitative evaluation possible. Different from the subjective evaluation method, objective evaluation methods can usually be extended to a large image data set. Therefore, our image database is much larger than the one used in the South Florida benchmark, which contains only 28 real images.

\subsection{Line-approximation algorithm}

As mentioned in Section 2, we use a line-approximation algorithm to estimate the edge-direction information. In this way, the fragments fed into the boundary-grouping component are in the form of straight line segments. Line approximation, or line fitting, is a well-studied problem with many effective methods available. While these methods 
differ in the mathematical formulations and the algorithmic solutions, the underlying basic ideas are the same: finding a set of line segments that are well aligned with the detected edges pixels. A key parameter in the line approximation is the dislocation-tolerance threshold $\delta_{t}$, which is the preassigned allowed discrepancy (in pixels) between an edge pixel and its mapping in the resulting line segments. With this parameter, the line-approximation method can find a minimum number of line fragments to fit all the edges. In this paper, we use an implementation by Peter Kovesi for the line approximation. This is a Matlab code that can be downloaded from http://www.csse.uwa.edu.au/ pk/Research/MatlabFns/.

Note that this implementation is not developed based on any special edge detectors.

To achieve an objective edge-detection evaluation, we need to consider the influence of selecting different $\delta_{t}$ 's on the boundary-detection performance. Clearly, a smaller $\delta_{t}$ will generate more shorter line fragments and a larger $\delta_{t}$ will generate fewer longer line fragments. In Section 5.1, we will conduct an empirical study on the influence of $\delta_{t}$. From this empirical study, we find that, regardless of the adopted edgedetectors and detector parameters, the same $\delta_{t}$ always provides us the best boundary-detection performance. Therefore, we can fix the parameter $\delta_{t}$ (and the line-approximation component) in the edge-detection evaluation.

\subsection{Ratio-contour algorithm}

In this paper, we use the ratio-contour algorithm [17] to implement the boundary-grouping component. In this algorithm, boundary saliency is measured by an unbiased combination of three important Gestalt laws: closure, proximity, and continuity, which have been verified by many previous psychological and psychophysical studies. Specifically, closure requires the boundary to be complete. Proximity requires the gap between two neighboring fragments to be small. Continuity requires the resulting boundary to be smooth. The ratio-contour algorithm always detects the global optimal boundary in terms of its boundary-saliency measure.

To achieve closed boundaries, we construct a set of smooth curve segments, as shown by the dashed curves in Figure 4(b), to connect the constructed fragments. Those dashed curves are another set of fragments. To distinguish them from the initial straight-line fragments, we call them virtual fragments and call the initial ones real fragments. Considering the boundary smoothness, the virtual fragments are constructed in such a way that each of them interpolates two real-fragment endpoints in $G^{1}$-continuity, that is, continuous locations and continuous tangent directions [22], as shown in Figure 4(c). Various gap-filling algorithms can be used for constructing the smooth virtual fragments, and in this paper, we use the Bezier-curve splines to construct them.

Ideally, we need to construct virtual fragments between each possible pair of fragment endpoints, as shown in Figure 4(c). In practice, however, we only construct virtual fragments that are likely to be along a salient closed boundary, as shown in Figure 4(b). A detailed discussion on this can be found in [17]. Based on the constructed real/virtual fragments, a valid closed boundary can be defined as a cycle that traverses a subset of real fragments and virtual fragments alternately, as shown in Figure 4(d). The goal of the boundary grouping is then to find from all such valid closed boundaries the one that has the largest perceptual saliency. Let $\mathbf{v}(t)=(x(t), y(t)), t \in[0, L(\mathbf{v})]$, be the arc-length parameterized representation [23] of a valid closed boundary, that is, $\mathbf{v}(L(\mathbf{v}))=\mathbf{v}(0)$, where $L(\mathbf{v})$ is the boundary length. In the ratio-contour algorithm [17], the cost (negatively related to the saliency) of this boundary is defined by

$$
R(\mathbf{v})=\frac{\int_{0}^{L(\mathbf{v})}\left[\sigma(t)+\lambda \cdot \kappa^{2}(t)\right] d t}{L(\mathbf{v})}
$$

where $\sigma(t)=1$ if $\mathbf{v}(t)$ is on a gap-filling virtual fragment and $\sigma(t)=0$, otherwise. $\kappa(t)$ is the curvature of the boundary at $\mathbf{v}(t)$.

In the numerator of (1), the first term $\int_{0}^{L(\mathbf{v})} \sigma(t) d t$ makes it biased towards a boundary with longer real fragments and shorter virtual fragments. This reflects the preference for better proximity. The second term $\int_{0}^{L(\mathbf{v})} \kappa^{2}(t) d t$ reflects the favor of smoother boundaries, or better continuity. The denominator normalizes the cost by the boundary length $L(\mathbf{v})$ to avoid a bias to shorter boundaries. $\lambda>0$ is a regularization factor that balances the proximity and continuity in the cost function. Boundary closure is included as the hard constraint in this algorithm: it only searches for closed boundaries. In [17], a graph-theoretic algorithm is developed to find the optimal closed boundary that globally minimizes the cost (1). We can see that the ratio-contour algorithm well integrates the properties of proximity, continuity, and closure into boundary grouping.

Several facts make the ratio-contour algorithm an appropriate choice for the boundary-grouping component in our evaluation framework. First, boundary grouping itself is a very challenging problem and so far, only a few algorithms can achieve closed boundaries from fragments. A comparison study in [17] has shown that the ratio-contour algorithm usually has a better performance than the prior stateof-the-art boundary-grouping methods. Second, both theoretical and experimental study in [17] has shown that the ratio-contour algorithm is able to detect the salient closed boundary from noisy background if the edge-detection step extract sufficient boundary features. Particularly, in [17], the ratio-contour algorithm was tested on a large set of synthetic data that mix the fragments from the sampled ground-truth boundary and noise and a very high boundarydetection accuracy was reported. Finally, the ratio-contour algorithm finds the globally optimal boundary in terms of its boundary-saliency measure and does not require any subjective or heuristic initialization. This preserves the objectivity of our edge-detection evaluation framework.

\subsection{Performance measure}

As discussed in Section 1, boundary detection can be regarded as a general-purpose application that functions like a bridge linking low-level edge detection to many high-level 


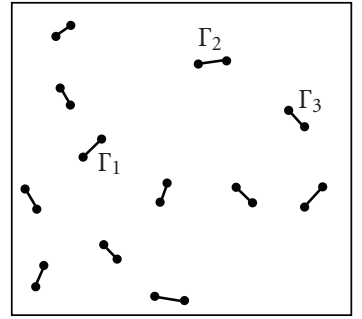

(a)

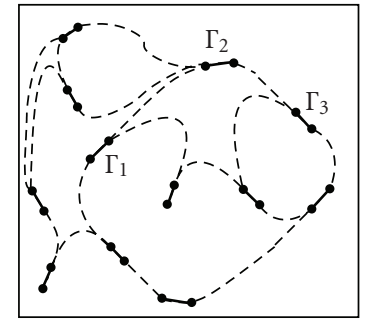

(b)

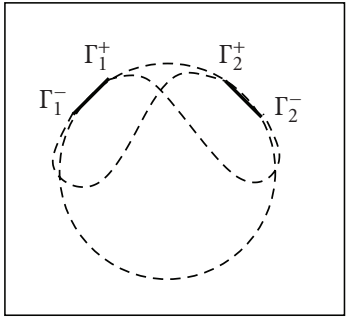

(c)

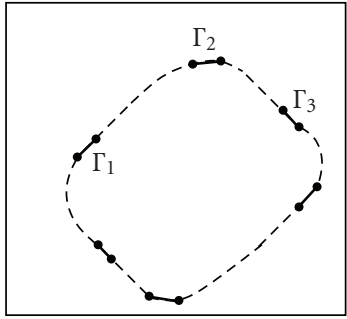

(d)

FIGURE 4: An illustration of the boundary grouping using the ratio-contour algorithm. (a) Straight-line fragments constructed from the edge-detection results. (b) Filling gaps between each pair of real-fragment endpoints with $G^{1}$-continuity. (c) Between each pair of real fragments, there are four possible gaps to fill without considering filling the gap between the two endpoints of the same real fragment. (d) The closed boundary extracted using the ratio-contour algorithm.

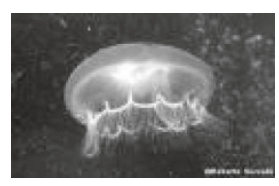

(a)

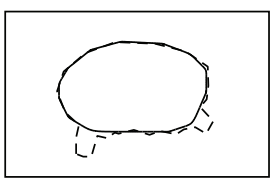

(b)

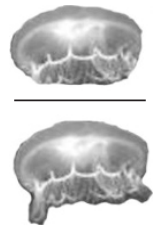

(c)
FIgURE 5: An illustration of the region-based performance measure. (a) Original image; (b) the ground-truth boundary (dashed curve) and the detected boundary (solid curve); (c) the performance measure $|A \cap B| /|A \cup B|$.

applications. The coincidence between the detected boundary and the ground-truth boundary reflects the performance of the adopted edge detection. If we measure the coincidence of these two boundaries in terms of two smooth curves, the resulting measure would be sensitive to the construction of the ground-truth boundary: even a small error there may introduce a larger error to the performance evaluation. In this paper, we adopt a region-based measure to accomplish this goal. Each image is a priori known to have a single salient closed boundary. Let region $A$ represent the ground-truth salient object. We perform an edge detection (with certain edge detector and certain detector parameters) on this image and then use the ratio-contour algorithm to detect a salient closed boundary, which, in fact, generates a region $B$ for the estimated salient foreground object. Denote the image as $I$, the edge detector as $\ell$, and the detector parameters as $\mu$. As illustrated in Figure 5, we measure the edge-detection

$$
P(I, \ell, \mu)=\frac{|A \cap B|}{|A \cup B|}=\frac{|A \cap B|}{|A|+|B|-|A \cap B|},
$$

where $|\cdot|$ is the operation of computing the region area.

The numerator, $|A \cap B|$, measures how much the true object region is detected. The denominator, $|A \cup B|$, is a normalization factor which normalizes the performance measure to the range of $[0,1]$. A performance of 1 is achieved if and only if the detected boundary completely coincides with the ground-truth boundary, that is, $A=B$. Zero performance indicates that there is no region-intersection between the detected object and the ground-truth object. With this normalization factor, the performance measure penalizes mistakenly detected regions (false positives). It is easy to see that this region-based measure is insensitive to small variations of the ground-truth boundary. This definition of the performance measure well incorporates the accuracy and recall measurement into one unified function, enabling quantitative, objective, and less computational intensive evaluation.

\section{EDGE-DETECTORS SELECTED FOR EVALUATION}

Considering both classical and recently-reported edge-detection methods, we chose five edge detectors: Sobel [9], LoG [18], Canny [3], Rothwell [19], and Edison [20] for evaluation. Each detector has its own parameter settings. In this paper, we evaluate not only different edge detectors, but also different parameter settings. Samples of edge-detection results using these five edge detectors are demonstrated in Figure 6. In this paper, we use the image-processing toolbox functions in Matlab for the Sobel, LoG, and Canny edge detectors. The Rothwell edge detector source code was downloaded from the $\mathrm{ftp}$ site of the South Florida Computer Vision Group (ftp://figment.csee.usf.edu/ pub/Edge_Comparison/source_code/) and the Edison edge detector was downloaded from the author's web page at http://www.caip.rutgers.edu/riul/research/code.html. In this section, we briefly describe these five edge detectors and their parameters.

\subsection{Sobel edge detector}

The Sobel edge detector [9] is one of the earliest edge detection methods. For many applications, it is used as a standard gradient computation method to retrieve the image gradient and edges. More specifically, the Sobel edge detector contains two directional filters:

$$
G_{x}=\left[\begin{array}{lll}
-1 & 0 & 1 \\
-2 & 0 & 2 \\
-1 & 0 & 1
\end{array}\right], \quad G_{y}=\left[\begin{array}{ccc}
-1 & -2 & -1 \\
0 & 0 & 0 \\
1 & 2 & 1 .
\end{array}\right] .
$$

These two filters convolve with the image separately to retrieve the image-gradient components along horizontal and 
vertical directions, respectively. Combining these two imagegradient components, the gradient magnitude is derived as $|\nabla I(x, y)|=\sqrt{\left(G_{x} * I\right)^{2}+\left(G_{y} * I\right)^{2}}$, where $*$ stands for the signal-convolution operation. From gradient magnitude to edges, a threshold $\delta_{s}$ is applied to find edge pixels. This introduces an intrinsic difficulty in Sobel edge detection (and also in many other edge detectors), that is, how to select the best threshold and how sensitive the threshold is in terms of overall performance. The Matlab implementation we used provides a default dynamic threshold $\hat{\delta}_{s}$. In our evaluation, we test different thresholds $\delta_{s}=p_{s} \hat{\delta}_{s}$ by varying the scaling factor $p_{s}$ in the range of $[0.5,1.5]$, that is, $p_{s}$ is the only parameter in evaluating the Sobel detector.

\subsection{LoG edge detector}

As first introduced in [18], the LoG (Laplacian of Gaussian) edge detector is a well-known method that exploits the second derivatives of pixel intensity to locate edges. The definition of a LoG filter actually is a combination of a Laplacian operator and a Gaussian filter:

$$
\nabla^{2} G_{\sigma}=\left(\frac{\partial^{2}}{\partial x^{2}}+\frac{\partial^{2}}{\partial y^{2}}\right) G_{\sigma}
$$

where a $2 \mathrm{D}$ symmetric Gaussian smoothing filter $G_{\sigma}$ is defined by

$$
G_{\sigma}(x, y)=\frac{1}{2 \pi \sigma^{2}} \exp \left\{-\frac{\left(x^{2}+y^{2}\right)}{2 \sigma^{2}}\right\} .
$$

In this edge detector, edges are detected by combining the information of the image-gradient magnitude and the zerocrossing points in the second-derivative map. One threshold $\delta_{L}$ is critical for LoG edge detector: only when the pixel (a) is a zero-crossing point in the second-derivative map, and (b) has a gradient magnitude large than $\delta_{L}$, do we select it as an edge pixel. Similar to Sobel, we treat $\delta_{L}$ as a scaled version of the default value provided in the LOG implementation in Matlab, and vary the scaling factor $p_{L}$ in the range of $[0.5,2.5]$. In another word, $p_{L}$ is the only detector parameter for LOG in our evaluation.

\subsection{Canny edge detector}

The Canny edge detector [3] is one of the most widely used edge detectors in computer-vision and image-processing community. In many applications, The Canny edge detector has been used as the standard image preprocessing technique. The Canny edge detector was shown to be superior to Sobel detector by subjective visual evaluation in [11]. In this paper, we include the Canny edge detector to see whether it does have more favorable performance in an objective and general boundary-detection framework. Canny edge detection consists of four steps: noise suppression, gradient computation, non-maximal suppression, and hysteresis. The first two steps are the same as the ones used in the Sobel edge detector. In the non-maximal suppression, edge pixel and edge direction are estimated by checking and tracing the neighboring pixels around pixels with large gradient magnitude. In the hysteresis, a high threshold $\delta_{\text {high }}$ and a low threshold $\delta_{\text {low }}$ are applied to remove spurious edges: it locates the first edge pixel by requiring its gradient magnitude to be larger than $\delta_{\text {high }}$ and then traces the following edge pixels by requiring the gradient magnitude to be larger than $\delta_{\text {low }}$. The unique feature of the Canny edge detector is its hysteresis step with a two-threshold operation. Usually, $\delta_{\text {high }}$ helps remove false positives and $\delta_{\text {low }}$ helps improve the edge-location accuracy. In general, the Canny edge detector has a tendency to detect long edges, which usually improves its performance in subjective evaluations. Similar to Sobel and Canny, we set $\delta_{\text {low }}=p_{c} \hat{\delta}_{\text {low }}$ and $\delta_{\text {high }}=p_{c} \hat{\delta}_{\text {high }}$ in our evaluation, where $\hat{\delta}_{\text {low }}$ and $\hat{\delta}_{\text {high }}$ are the defaults provided in Matlab. Therefore, the scaling factor $p_{c}$ is the only detector parameter and we also vary it in the range of $[0.5,2.5]$ in our evaluation.

\subsection{Rothwell edge detector}

Many edge detectors, including the Canny edge detector, perform poorly at edge junctions and corners. As explained in [24], this is mainly caused by the difficulty in estimating the correct direction information at edge junctions and corners. Consequently, edge detection usually produces incorrect or incomplete topology around corners and junctions. The Rothwell edge detector [19] can partially address this problem in maintaining the scene topology of images. Similar to the Canny edge detector, the Rothwell edge detector applies Gaussian smoothing first to reduce image noise and then computes the gradient magnitude and direction. Unlike the Canny edge detector, the Rothwell edge detector only uses the low threshold $\delta_{\text {low }}$ in hysteresis to filter spurious edges, while using another image-dependent dynamic threshold to further reduce the number of detected edges. With this dynamic threshold, it can detect edges with varied gradient magnitudes. It is indicated in [19] that the Rothwell edge detector has two advantages over other methods: subpixel accuracy of the detected edges and better performance at edge junctions. By choosing this detector into our evaluation, we expect to find whether these two advantages actually benefit the application of salient boundary detection. Since the Rothwell edge detector chooses its high threshold automatically, the only parameter in our evaluation is $p_{r}$ which controls the lower threshold $\delta_{\text {low }}$. We vary $p_{r}$ in the range of $[3,18]$, as suggested in the Rothewell implementation we used.

\subsection{Edison edge detector}

Developed by Meer and Georgescu [20], the Edison edge detector not only detects edges, but also provides two confidence measures, $\eta$ and $\rho$, associated with each detected edge. These two confidence measures are expected to be further exploited in later high-level applications that use this edge detector as the first step for feature extraction. A templatematching approach is used in the Edison edge detector to 

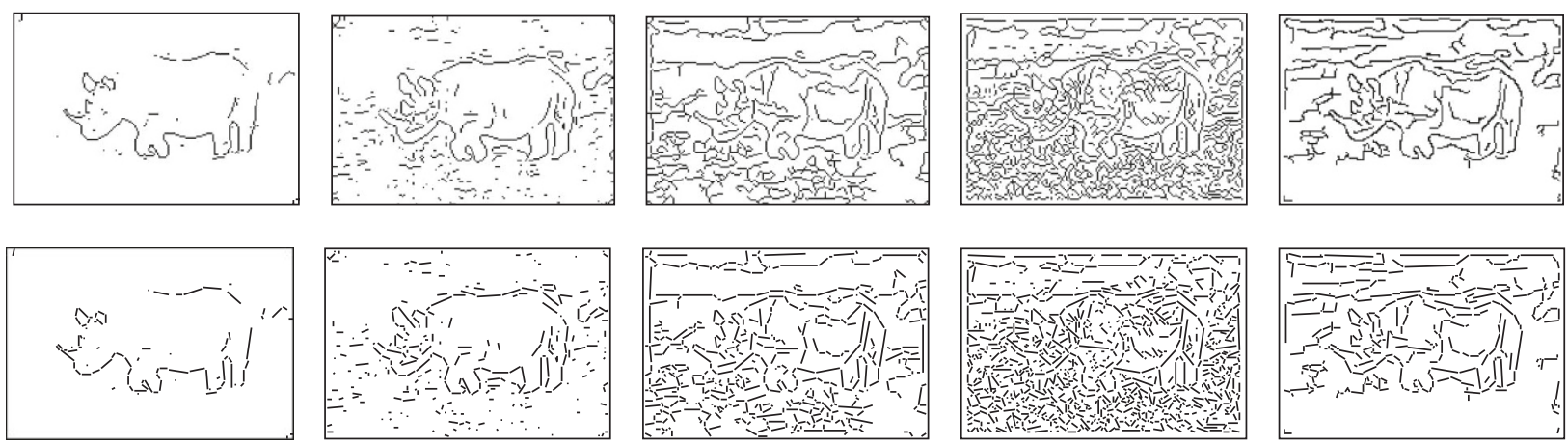

FIgure 6: Sample edge-detection and line approximation results. The original image is the first one shown in Figure 3. Top row shows edge-detection results from the five edge detectors with their default parameters. From left to right are the results from Sobel, LoG, Canny, Rothwell, and Edison, respectively. The bottom row shows the line-approximation results from the respective edge detection. See Table 1 for the default parameters of each edge detector.

derive the edge confidence $\eta$, which measures the correlation between the considered edge and an ideal edge template with the same gradient direction. The gradient-magnitude confidence $\rho$ is calculated by counting the percentage of pixels that have a gradient magnitude less than that of the considered edge. Both confidence measures take values in the range of $[0,1]$. In general, the Edison edge detector uses an approach similar to Canny to locate edge pixels and the major difference lies in that the Edison edge detector incorporates these two confidence measures in the hysteresis step. In the Edison edge detector, two decision planes $f^{(L)}(\eta, \rho)$ and $f^{(H)}(\eta, \rho)$, which are determined by the confidence measures $\eta$ and $\rho$, are calculated to replace the two fixed thresholds in Canny detector. In [20], it is claimed that these two decision planes introduce more flexibility and robustness to the edge detection. The Edison edge detector contains the maximum number ( 9 in total) of free parameters among all edge detectors. Obviously, it is neither possible nor necessary to exhaustively evaluate all of them. In our evaluation, we use the "boxed" decision planes and evaluate two most important parameters, $p_{e}^{H}$ and $p_{e}^{L}$, where $p_{e}^{H}=\eta_{\text {high }}=\rho_{\text {high }}$ and $p_{e}^{L}=\eta_{\text {low }}=\rho_{\text {low }}$. These two parameters determine the thresholds of confidence measures in the decision planes and we varied them in the range of $[0.6,1]$ in our evaluation.

\section{PERFORMANCE EVALUATION}

\subsection{Line-approximation parameter selection}

As discussed in Section 3.2, line approximation is the middle step in our evaluation framework. Therefore we need to carefully select the line-approximation settings in order to compare edge detectors fairly. As mentioned in Section 3.2, the line-approximation algorithm has an important dislocation-tolerance parameter $\delta_{t}$ which gives the maximal distance allowed for one edge pixel to be included in an approximated line segment. A small $\delta_{t}$ generates many short fragments while a large one may produce long fragments that are not very well aligned with edge pixels.
First, we conducted experiments to show the sensitivity of $\delta_{t}$ to boundary detection. The results are shown in Figure 7, which, together with all the other performance figures in Section 5, shows cumulative-performance histogram curve, which describe the performance distribution on all 1030 images. As shown in Figure 7, the $x$-axis represents the percentage of images, and the $y$-axis indicates the performance defined in Section 3.4. A data point $(x, y)$ along a curve indicates that, under this specified setting, $100 \cdot x$ percent of the images produce boundaries with an accuracy lower than $y$ in terms of the given ground-truth boundaries. Equivalently, this also means that $100 \cdot(1-x)$ percent of the images produce boundaries with performance better than $y$. Any change in the setting of edge-detection, line-approximation, or boundary-grouping components will produce a new performance curve for that setting. Obviously, the setting $\alpha$ achieves better performance than the setting $\beta$ if the performance curve of $\alpha$ is above that of $\beta$ in the cumulative-performance figure.

We varied $\delta_{t}$ in the line approximation for the five different edge detectors and some results are shown in Figure 7. This experiment was also conducted for many other detector-parameter settings, and just like the examples shown in Figure 7, all these experiments show that $\delta_{t}=1$ almost always produces the best performance for all the five detectors. Thus we conclude that this optimal value is largely uncorrelated to the edge detectors and the detector parameters. For our evaluation, we simply choose $\delta_{t}=1$ in line approximation for all of the remaining experiments.

In fact, we also see from Figure 7 that the boundarydetection performance does not degrade much by choosing a $\delta_{t} \in[0.5,2]$. To some extent, this indicates that the exact alignment between all edge pixels and the ground-truth boundary is not necessarily critical for boundary detection. In another word, in general-purpose boundary detection, there is no obvious advantage of introducing subpixel accuracy in edge detection. However, we do see that, when $\delta_{t}$ is very high, say more than 4 pixels, the boundary-detection performance degrades significantly. This shows that, if the line segments are estimated at a very coarse level, there is a 
TABLE 1: A summary of the edge detectors and their detector parameters that are evaluated in Figure 8 . The numbers with “*” are the bestaverage-performance parameters, and the numbers in bold face are the default parameters used in the implementations. Since Rothwell and Edison softwares provide no default parameters, we use their best-average-performance parameters as default ones.

\begin{tabular}{llll}
\hline Detector & Parameter & Figure 8 & Parameters evaluated \\
\hline Sobel & $p_{s}$ & (a) & $\left\{0.5,0.75, \mathbf{1}^{*}, 1.25,1.5\right\}$ \\
LoG & $p_{L}$ & (b) & $\left\{0.5,0.75, \mathbf{1}, 1.5,2^{*}, 2.5\right\}$ \\
Canny & $p_{c}$ & (c) & $\left\{0.5,0.75, \mathbf{1}, 1.5,2^{*}, 2.5\right\}$ \\
Rothwell & $p_{r}$ & (d) & $\left\{4,7,10,13, \mathbf{1 6}^{*}\right\}$ \\
Edison & $p_{e}^{L}-p_{e}^{H}$ & (e) & $\{0.6-0.75,0.6-0.8,0.6-0.9,0.6-0.93, \mathbf{0 . 6}-\mathbf{0 . 9 7 *}, 0.6-0.99\}$ \\
\hline
\end{tabular}

large discrepancy from the ground truth boundary, which, consequently, seriously reduces the boundary detection performance.

\subsection{Edge-detector performance}

In this subsection, we conducted experiments to evaluate the performance of the five edge detectors described in Section 4 . First, we evaluated each edge detector under different parameter settings to investigate its sensitivity and optimality in terms of the detector parameters. The tested parameter settings for each detector are summarized in Table 1 and the cumulative-performance histogram curves of each detector under various parameter settings are shown in Figure 8. The performance in Figure 8 is derived from the experiments on all the images in our database.

In Figure 8, we also show an "optimal"-performance curve for each edge detector (the curve with the symbol “*”). This represents the performance of each detector if we can dynamically find and apply the optimal parameter setting for each image. ${ }^{1}$ More specifically, let $\ell_{i}$ represent the $i$ th edge detector, and $\mu_{i j}$ represent the $j$ th parameter setting of $\ell_{i}$. The performance of $\ell_{i}$ on the $n$th image $I_{n}$ with parameter $\mu_{i j}$ is then $P\left(I_{n}, \ell_{i}, \mu_{i j}\right)$, as defined in (2). The "optimal" performance of edge detector $\ell_{i}$ on the image $I_{n}$ is defined as

$$
P_{\text {optimal }}\left(I_{n}, \ell_{i}\right)=\max _{j}\left\{P\left(I_{n}, \ell_{i}, \mu_{i j}\right)\right\}
$$

The optimal-performance curve in Figure 8, to some extent, gives an upper-bound of the potential performance of an edge detector by varying parameters for each image.

Certainly, finding the optimal detector parameters for each image is usually a difficult problem. One easier way is to use a constant detector parameter for each detector. The question is which parameter can lead to best performance on all images. In this paper, we define the best-averageperformance (BAP) parameters to model such best constant parameters. More specifically, for detector $\ell_{i}$ with parameter

\footnotetext{
${ }^{1}$ Strictly speaking, the "optimal" is only defined in terms of parameter spaces given in Table 1.
}

$\mu_{i j}$, the average performance on all $N$ images is

$$
\bar{P}\left(\ell_{i}, \mu_{i j}\right)=\frac{1}{N} \sum_{n} P\left(I_{n}, \ell_{i}, \mu_{i j}\right),
$$

and the BAP parameter is $\mu_{i j}$ with

$$
j^{*}=\arg \max _{j} \bar{P}\left(\ell_{i}, \mu_{i j}\right) .
$$

Obviously, for any image $I_{n}$ in the database, $P\left(I_{n}, \ell_{i}, \mu_{i j *}\right) \leq$ $P_{\text {optimal }}\left(I_{n}, \ell_{i}\right)$. The numbers in Table 1 with the symbol " $*$ " indicate the BAP parameters for the five selected detectors.

The Edison edge detector has two parameters, $p_{e}^{L}$ and $p_{e}^{H}$, which may substantially increase its parameter space in our evaluation. However, we find that when $p_{e}^{H}$ is fixed, $p_{e}^{L}$ has little effect on performance, as shown in Figure 9. Therefore, we only vary parameter $p_{e}^{H}$ for Edison detector in the evaluation and the result is shown in Figure 8(e).

From Figure 8, we have the following observations. First, detector-parameter selection has a large impact on the final performance. In fact, for all five edge detectors, varying the selected parameters usually results in significantly different boundary-detection performance. Second, the default edgedetector parameters in Matlab may not be optimal in terms of the proposed evaluation framework. For example, the performance of Canny detector is significantly improved by setting $p_{c}=2$, that is, increasing the default thresholds by a factor of 2 . Third, for all five selected detectors, the performance with a fixed parameter is far below the optimal performance. This indicates that there is a considerable scope for performance improvement by dynamic parameter selection, that is, finding the optimal parameter for each individual image.

To compare the relative performance of different edge detectors, we simply count the number of images on which one detector outperforms the other four. For example, if edge detector $\ell_{i}$ achieves the best performance on the image $I_{n}$, we consider $\ell_{i}$ the winner on $I_{n}$. We then count the number of winning images of each edge detector for comparison. To make the comparison fairer, we choose the BAP parameter (as indicated in Table 1) for each detector. The number of winning images of each edge detector is given in Table 2. The "performance constraint" column in Table 2 shows the threshold for a data-selection process, which excludes the images with a winning performance that does not satisfy this constraint. For example, in the row with a performance constraint " $>0.75$ ", the images are counted only 
TABLE 2: The number of winning images of each detector with different performance constraints.

\begin{tabular}{lcccccc}
\hline Performance constraint & Sobel & LoG & Canny & Rothwell & Edison & Total no. of images \\
\hline$>0$ & 230 & 184 & 213 & 219 & 184 & 1030 \\
$>0.35$ & 189 & 145 & 177 & 180 & 131 & 161 \\
$>0.55$ & 140 & 108 & 128 & 73 & 129 & 636 \\
$>0.75$ & 70 & 45 & 73 & 33 & 40 & 335 \\
$>0.90$ & 24 & 9 & 27 & & 40 & 133 \\
\hline
\end{tabular}

when the winning performance is larger than 0.75. From Table 2, we can see that Sobel, Canny, and Rothwell have a similar performance, while LoG does not perform quite as well. However, the difference is not significant among these five detectors. Particularly, for the images in which the boundary-detection accuracy is high (e.g., the row with the performance-constraint “ $>0.75$ ”), Edison performs as well as Sobel, Canny, and Rothwell.

\subsection{Combination of edge detectors}

Beside evaluating and comparing the performance of individual edge detectors, it is also important to know whether and how these edge detectors are statistically related. If these five detectors can complement each other in edge detection, then it would be worthwhile to investigate ways to boost the performance by combining them. To better understand the correlation of these five edge detectors, we introduce a virtual combined detector, in which the winning edge detector for each image is used to process this image. We name the performance of such a virtual detector as combined performance:

$$
P_{\text {combined }}\left(I_{n}, \boldsymbol{\mu}\right)=\max _{i}\left\{P\left(I_{n}, \ell_{i}, \mu_{i}\right)\right\}
$$

Note that in the combined detector, the parameters for each individual detector are fixed and preset, and are denoted by $\mu_{i}$ for detector $\ell_{i}$, and in (9), $\boldsymbol{\mu}=\left\{\mu_{i}, i=1,2, \ldots, 5\right\}$ is the set consisting of the five fixed parameters. This combined performance gives the upper-bound performance by switching edge detectors (with fixed detector parameters) on each image.

Figure 10 shows the performance of all five detectors (with BAP parameters), their respective optimal performance, and the combined performance using the BAP parameters (labelled as "combined"). Again, we first see that these five edge detectors have similar performance with their BAP parameters. This is consistent with the information provided in Table 2. In addition, Figure 10 shows an interesting result: switching edge detectors with BAP parameters for individual images can drastically improve the final performance, and the combined performance of these five edge detectors is even slightly better than the optimal performance of each individual edge detector. This clearly indicates that these five edge detectors can complement each other to get much better performance.
Figure 11 compares the combined performance when each detector uses its BAP parameter and the combined performance when each detector uses its default parameter. The result shows very close performance between them. Combining the results shown in Figure 10, we see that, even using only default parameters for each detector, we may still achieve highly improved edge-detection performance if we have a way to select a suitable detector for each image.

In Figure 10, we also show a curve of the "ideal" performance. This performance is obtained by finding the best possible performance through edge-detector switching and detector-parameter optimization for each individual image; that is,

$$
P_{\text {ideal }}\left(I_{n}\right)=\max _{i, j}\left\{P\left(I_{n}, \ell_{i}, \mu_{i j}\right)\right\}
$$

We can see that the ideal performance is much higher than the performance of each individual edge detector. This tells us that, without developing new edge detectors, if we can find suitable detector and detector parameters for each image, we can get much better performance than that provided by any current individual detector.

In Figure 10, we can find that, even using a detector with the ideal performance, there is still a significant portion (20\%-40\%) of images with low performance. This may be a result of the boundary-grouping component. It is a recognized fact that boundary grouping is a very challenging problem and a perfect boundary detection for any image is almost impossible. Yet this does not diminish the significance of our work since our main goal is to evaluate edge detection rather than boundary grouping. To further justify our evaluation results, we apply a performance constraint to exclude the low-performance images which are less discriminating in differentiating edge detector performance. This data selection is based on one assumption: if the boundary detection fails with all possible edge detectors and detector parameters, we can hardly judge which edge detector is better. But if some fail and some succeed, we can incorporate such data for comparison.

Following this strategy, we choose from our database a subset of 526 images that produce an ideal performance larger than 0.7 . The selected images still show good variety and complexity. On these images, we repeat the same experiments and the results are shown in Figure 12. We can see that, although all the performance curves are moved up, the relative locations among them are similar to those shown in 

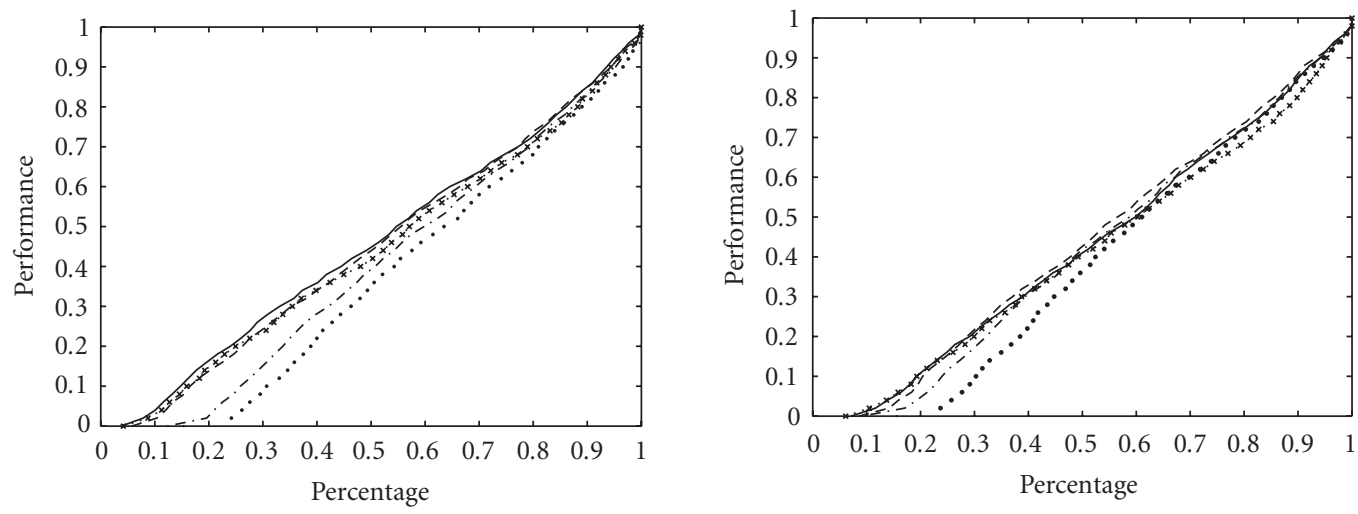

$$
\begin{aligned}
& \cdots \times \cdots \\
& --1 \\
& --2
\end{aligned}
$$$$
\cdot-3
$$

(a) Sobel

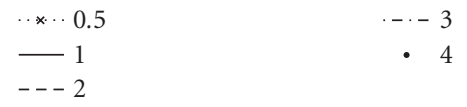

(b) LoG

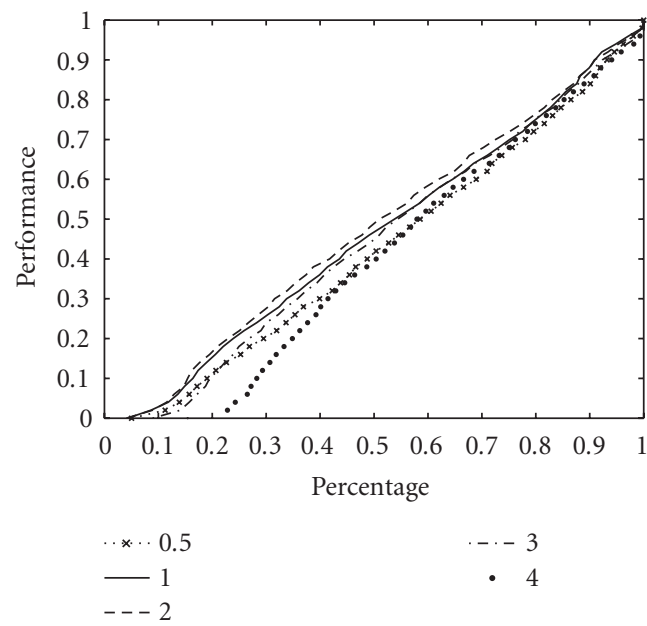

(c) Canny
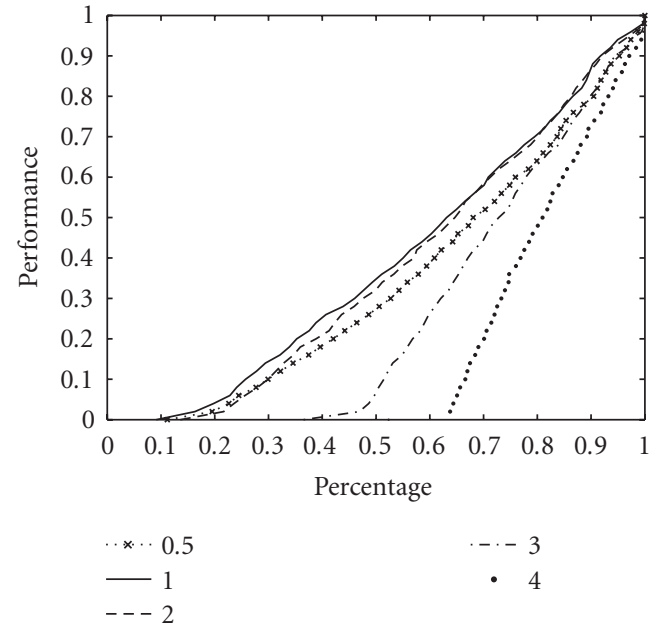

(d) Rothwell

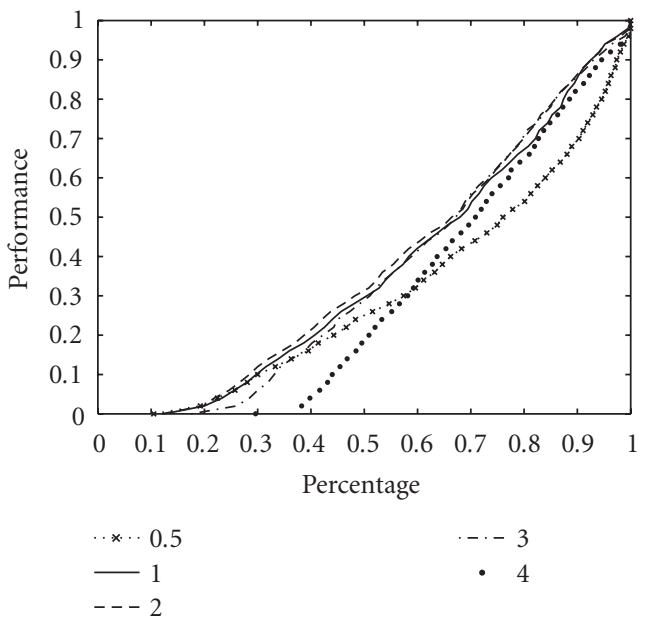

(e) Edison

FIGURE 7: Evaluation of the effect and the sensitivity of the dislocation-tolerance parameter $\delta_{t}$ in boundary detection. The parameter settings for these five edge detectors are (a) Sobel, $p_{s}=1$; (b) LoG, $p_{L}=1$; (c) Canny, $p_{c}=1$; (d) Rothwell, $p_{r}=16$; (e) Edison, $p_{e}^{H}=0.9, p_{e}^{L}=0.8$. 

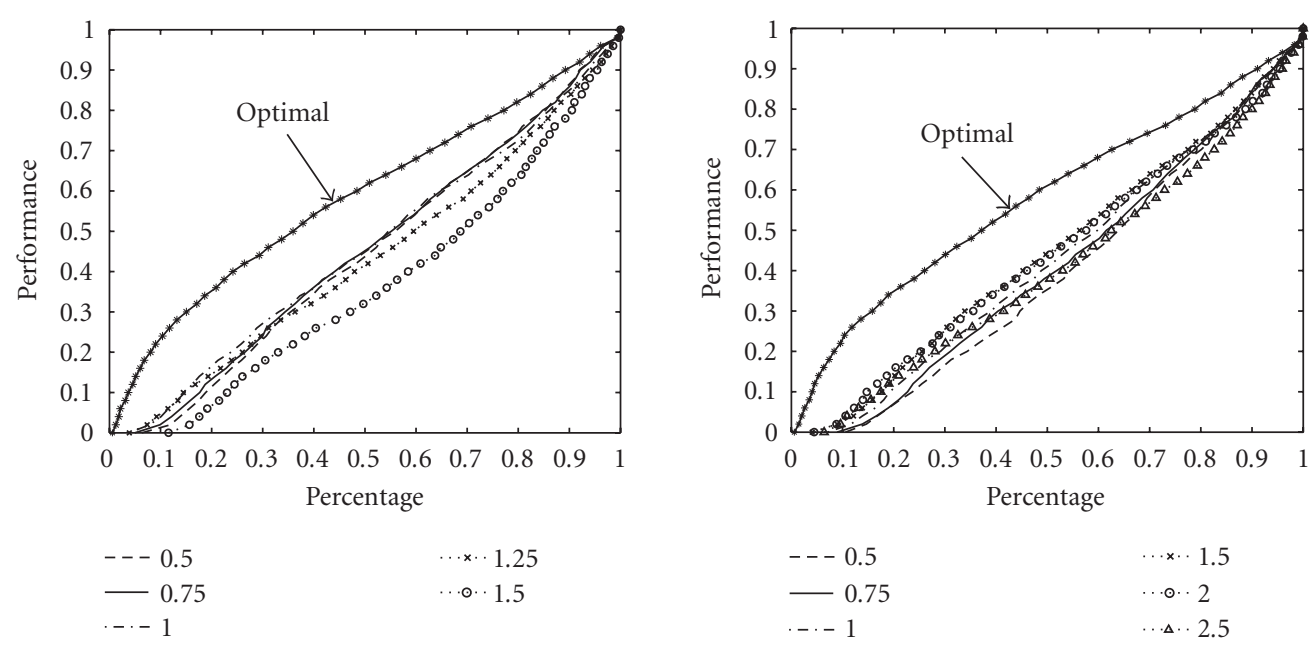

(a) Sobel

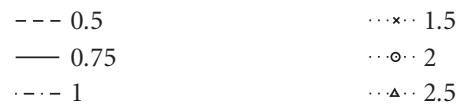

(b) LoG

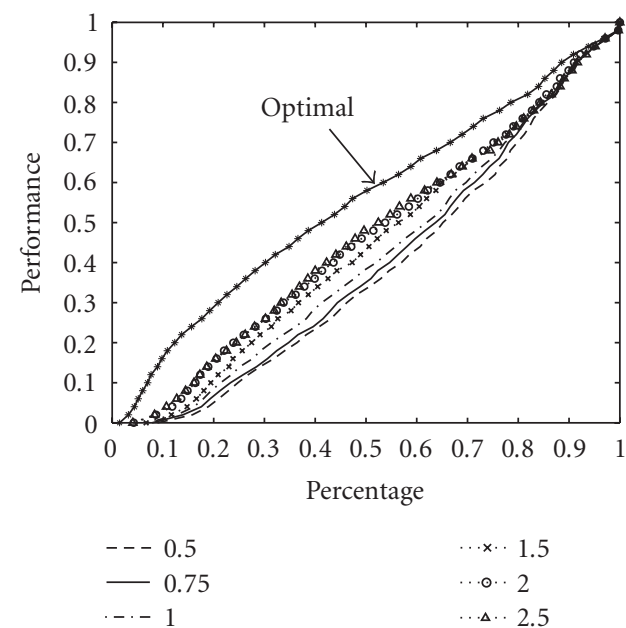

(c) Canny

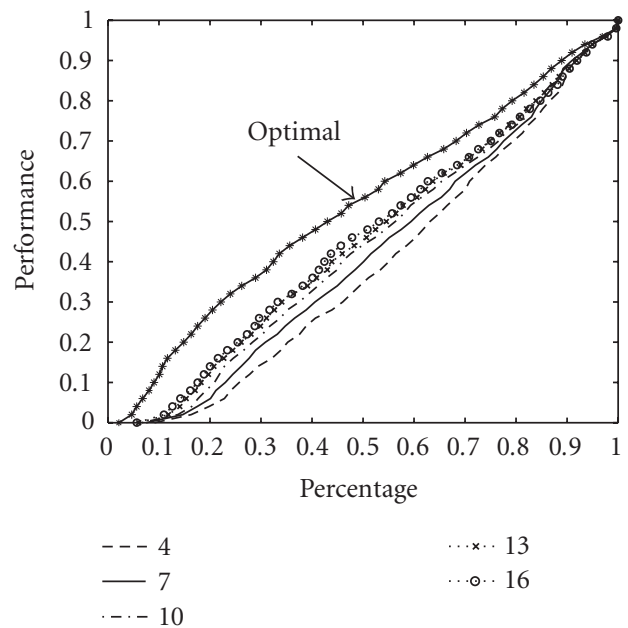

(d) Rothwell

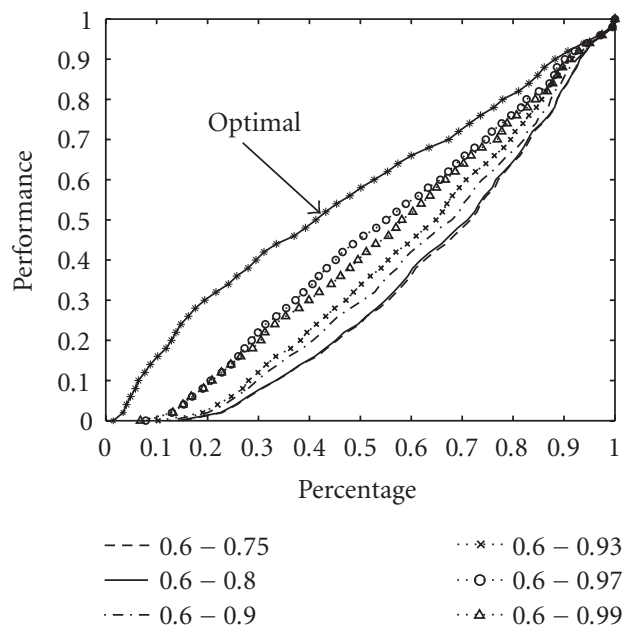

(e) Edison

FIGURE 8: Performance evaluation on each detector by varying detector parameters. (a) Sobel: varying $p_{s}$; (b) LoG: varying $p_{L}$; (c) Canny: varying $p_{c}$; (d) Rothwell: varying $p_{r}$; (e) Edison: varying $p_{e}^{L}-p_{e}^{H}$. 


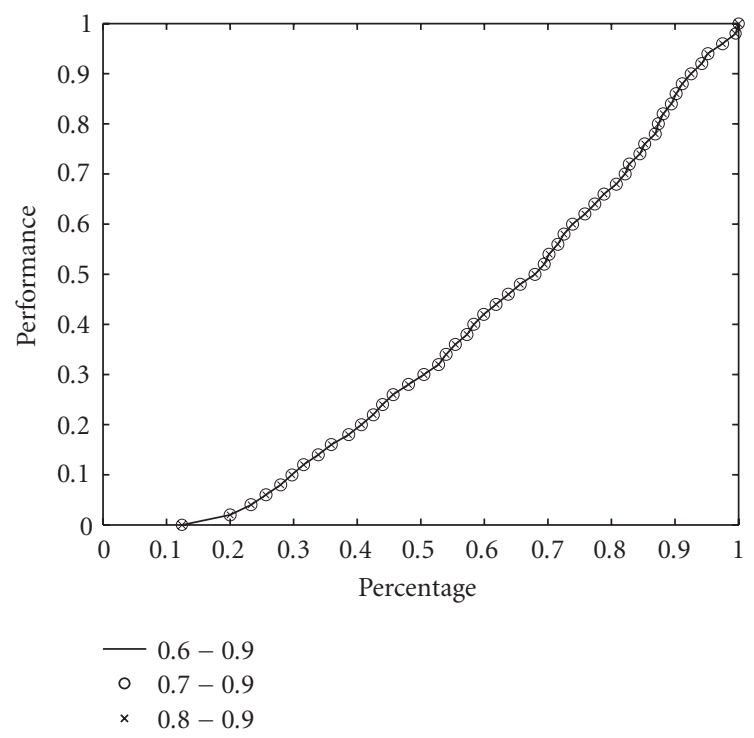

Figure 9: Performance evaluation on Edison detector by varying parameter $p_{e}^{L}$. Result indicates that $p_{e}^{L}$ has little effect on the performance.

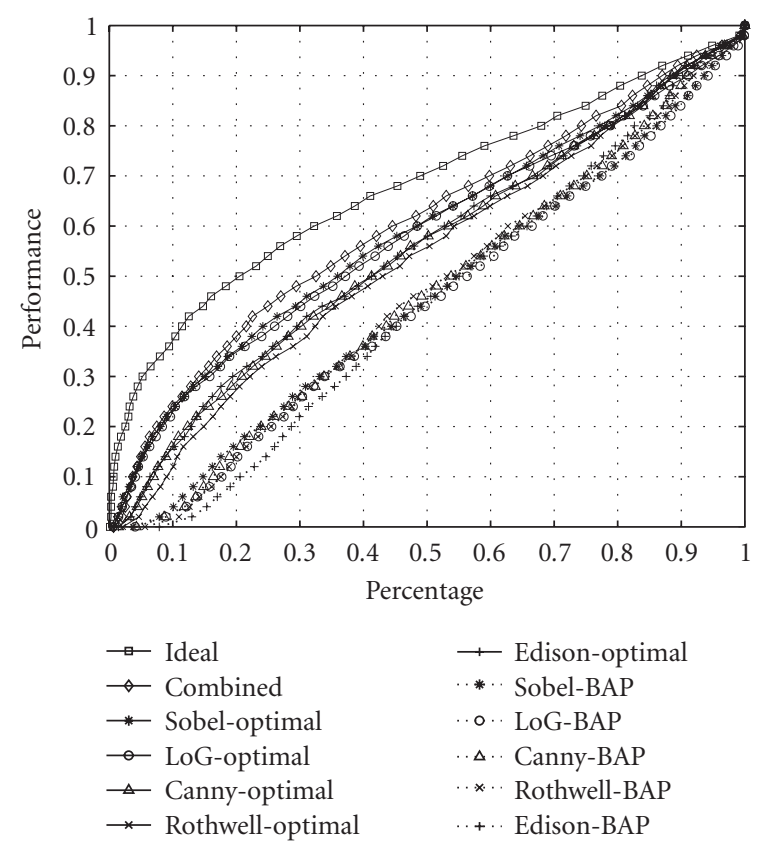

FIgURE 10: Edge-detection evaluation on all 1030 images in the database.

Figure 10. All the conclusions drawn from Figure 10 can also be drawn from Figure 12.

To summarize, we can draw the following conclusions from our performance-evaluation results.

(1) Line approximation is largely uncorrelated to the selected edge detectors in our evaluation framework. The lineapproximation threshold can be selected within a certain

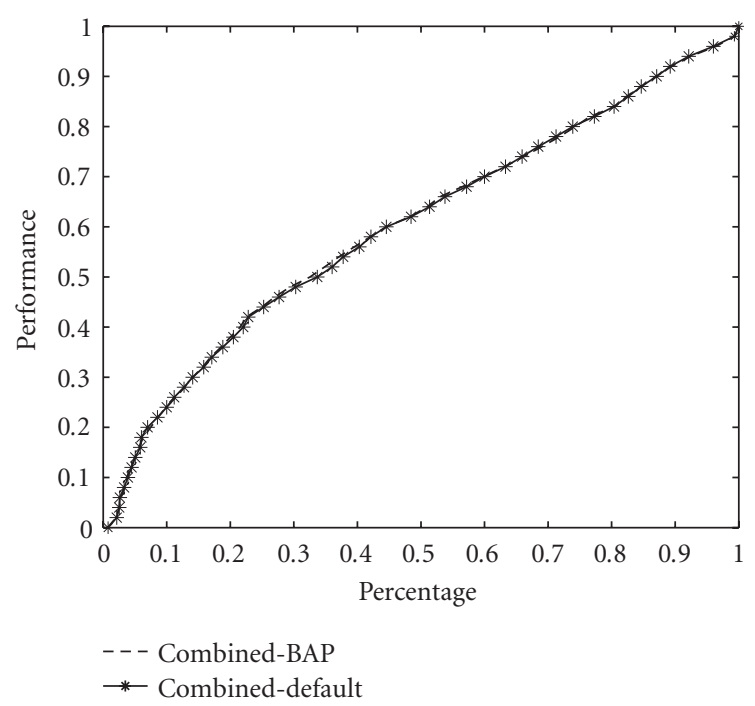

FIGURE 11: Comparison of the combined performance using default parameters ("combined-default") and that using BAP parameters ("combined-BAP").

range. Sub-pixel accuracy of edge detection is neither required nor necessary for salient boundary detection.

(2) The overall performance of the five edge detectors is very similar. The performance difference is marginal and there is no obvious winner on the collected images.

(3) The selection of the detector parameters has significant impact on the final performance. The default parameters used in Matlab are not optimal for some edge detectors in terms of boundary-detection performance.

(4) The evaluated edge detectors do complement each other. With the right way of combining them, the performance can be greatly improved.

(5) Even without introducing new edge detectors, there is still considerable scope for performance improvement by optimizing parameters and/or by selecting the edge detector according to the input image.

\section{CONCLUSION}

It is a very challenging problem to evaluate edge-detection results produced by various edge detectors with different detector parameters. In this paper, we presented new methodology and a framework for evaluating edge detections by checking the likelihood of locating salient object boundaries from the detected edges. We collected a large data set of 1030 real images for evaluation. The ground truth boundaries were manually extracted and a quantitative performance measure was defined. The proposed evaluation method not only is objective thanks to the unambiguous ground truth, but also has enough generality to be useful for other computer-vision and image-processing applications. In this paper, we compared and evaluated five classical and recent edge detectors: Sobel, LoG, Canny, Rothwell, and Edison under various parameter settings. 


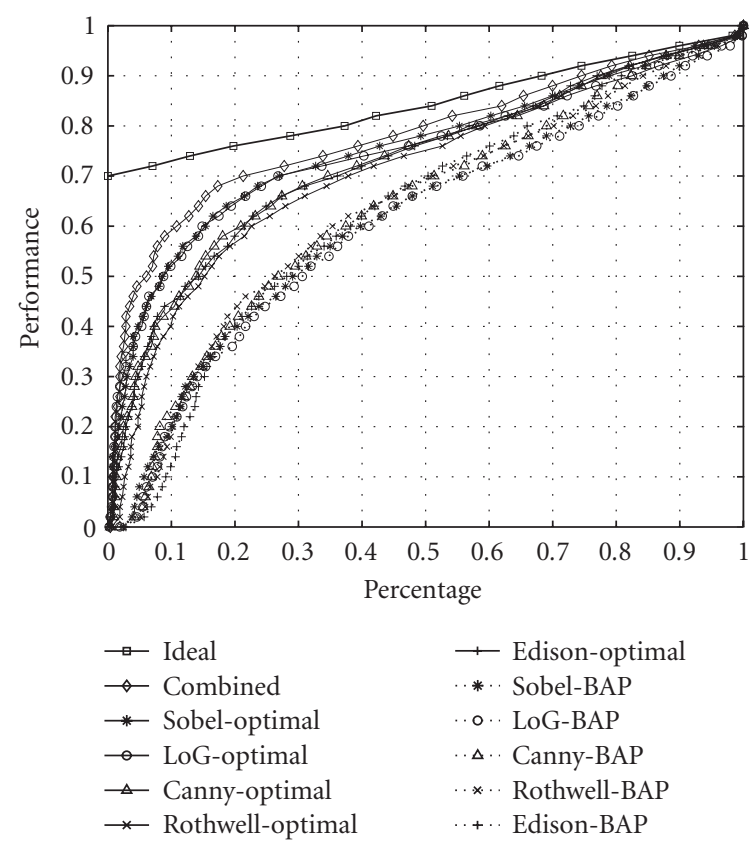

Figure 12: Edge-detection evaluation on the selected 526 images with the ideal performance larger than 0.7 .

The performance evaluation results show that the performance of these edge detectors is very similar but the default parameters in these edge detectors are far from the best for boundary detection. There is considerable scope for performance improvement by introducing better dynamic parameter selection. We also found that these edge detectors complement each other and an appropriate combination of them may improve the boundary-detection performance significantly. These findings naturally lead to two possible future research directions: improving image-dependent detector-parameter selection and boosting performance with a combination of edge detectors.

\section{ACKNOWLEDGMENTS}

We would like to thank the anonymous reviewers for their insightful suggestions. This work was funded, in part, by NSFEIA-0312861 and a grant from the University of South Carolina Research and Productivity Scholarship Fund.

\section{REFERENCES}

[1] B. Julesz, "A method of coding TV signals based on edge detection," Bell System Technology, vol. 38, no. 4, pp. 1001-1020, 1959.

[2] K. W. Bowyer, C. Kranenburg, and S. Dougherty, "Edge detector evaluation using empirical ROC curves," Computer Vision and Image Understanding, vol. 84, no. 1, pp. 77-103, 2001.

[3] J. Canny, "A computational approach to edge detection," IEEE Transactions on Pattern Analysis and Machine Intelligence, vol. 8, no. 6, pp. 679-698, 1986.

[4] L. A. Iverson and S. W. Zucker, "Logical/linear operators for image curves," IEEE Transactions on Pattern Analysis and Machine Intelligence, vol. 17, no. 10, pp. 982-996, 1995.
[5] S. Konishi, A. L. Yuille, J. M. Coughlan, and S. C. Zhu, "Statistical edge detection: learning and evaluating edge cues," IEEE Transactions on Pattern Analysis and Machine Intelligence, vol. 25, no. 1, pp. 57-74, 2003.

[6] J. M. S. Prewitt, "Object enhancement and extraction," in Picture Processing and Psychopictorics, B. S. Lipkin and A. Rosenfeld, Eds., pp. 75-149, Academic Press, New York, NY, USA, 1970.

[7] K. R. Rao and J. Ben-Arie, "Optimal edge detection using expansion matching and restoration," IEEE Transactions on Pattern Analysis and Machine Intelligence, vol. 16, no. 12, pp. 1169-1182, 1994.

[8] L. G. Roberts, "Machine perception of three-dimensional solids," in Optical and Electro-Optical Information Processing, J. T. Tippett, Ed., pp. 159-197, MIT Press, Cambridge, Mass, USA, 1965.

[9] I. E. Sobel, "Camera models and machine perception," Ph.D. dissertation, Stanford University, Stanford, Calif, USA, 1970.

[10] L. Kitchen and A. Rosenfeld, "Edge evaluation using local edge coherence," IEEE Transactions on Systems, Man, and Cybernetics, vol. 11, no. 9, pp. 597-605, 1981.

[11] M. D. Heath, S. Sarkar, T. Sanocki, and K. W. Bowyer, "A robust visual method for assessing the relative performance of edge-detection algorithms," IEEE Transactions on Pattern Analysis and Machine Intelligence, vol. 19, no. 12, pp. 13381359, 1997.

[12] S. Baker and S. K. Nayar, "Global measures of coherence for edge detector evaluation," in Proceedings of IEEE Computer Society Conference on Computer Vision and Pattern Recognition (CVPR '99), vol. 2, pp. 373-379, Fort Collins, Colo, USA, June 1999.

[13] P. L. Palmer, H. Dabis, and J. Kitler, "A performance measure for boundary detection algorithms," Computer Vision and Image Understanding, vol. 63, no. 3, pp. 476-494, 1996.

[14] Y. Yitzhaky and E. Peli, "A method for objective edge detection evaluation and detector parameter selection," IEEE Transactions on Pattern Analysis and Machine Intelligence, vol. 25, no. 8, pp. 1027-1033, 2003.

[15] Q. Zhu, "Efficient evaluations of edge connectivity and width uniformity," Image and Vision Computing, vol. 14, no. 1, pp. 21-34, 1996.

[16] X. Y. Jiang, A. Hoover, G. Jean-Baptiste, D. Goldgof, K. W. Bowyer, and H. Bunke, "A methodology for evaluating edge detection techniques for range images," in Proceedings of 2 nd Asian Conference on Computer Vision (ACCV'95), vol. 2, pp. 415-419, Singapore, December 1995.

[17] S. Wang, T. Kubota, J. M. Siskind, and J. Wang, "Salient closed boundary extraction with ratio contour," IEEE Transactions on Pattern Analysis and Machine Intelligence, vol. 27, no. 4, pp. 546-561, 2005.

[18] D. Marr and E. C. Hildreth, "A theory of edge detection," Proceedings of the Royal Society of London. Series B, vol. 207, no. 1167, pp. 187-217, 1980.

[19] C. A. Rothwell, J. L. Mundy, W. Hoffman, and V.-D. Nguyen, "Driving vision by topology," in Proceedings of IEEE International Symposium on Computer Vision (ISCV '95), pp. 395400, Coral Gables, Fla, USA, November 1995.

[20] P. Meer and B. Georgescu, "Edge detection with embedded confidence," IEEE Transactions on Pattern Analysis and Machine Intelligence, vol. 23, no. 12, pp. 1351-1365, 2001.

[21] D. Martin, C. Fowlkes, D. Tal, and J. Malik, "A database of human segmented natural images and its application to evaluating segmentation algorithms and measuring ecological statistics," in Proceedings of 8th IEEE International Conference on 
Computer Vision (ICCV '01), vol. 2, pp. 416-423, Vancouver, BC, Canada, July 2001.

[22] J. D. Foley, A. van Dam, S. K. Feiner, and J. F. Hughes, Computer Graphics: Principles and Practice in C, Addison Wesley, Reading, Mass, USA, 2nd edition, 1995.

[23] R. H. Bartels, J. C. Beatty, and B. A. Barsky, An Introduction to Splines for Use in Computer Graphics and Geometric Modelling, Morgan Kaufmann, Los Altos, Calif, USA, 1987.

[24] D. A. Forsyth and J. Ponce, Computer Vision: A Modern Approach, Prentice-Hall, Upper Saddle River, NJ, USA, 2003.

Song Wang received his Ph.D. degree in electrical and computer engineering from the University of Illinois at Urbana-Champaign in 2002. From 1998 to 2002, he also worked as a Research Assistant in the Image Formation and Processing Group of the Beckman Institute, University of Illinois at Urbana-Champaign. Since August 2002, he has been an Assistant Professor in the Department of Computer Science and Engi-

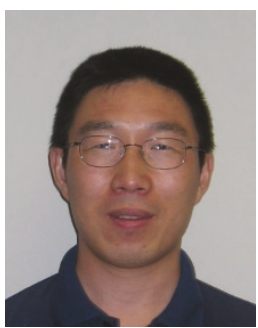
neering at the University of South Carolina. His research interests include computer vision, medical image processing, and machine learning.

Feng Ge received his B.S. degree from Tsinghua University, Beijing, in July 2002. Since August 2004, he has been a Master student in the Department of Computer Science and Engineering at the University of South Carolina. His research interests are computer vision and medical imaging.

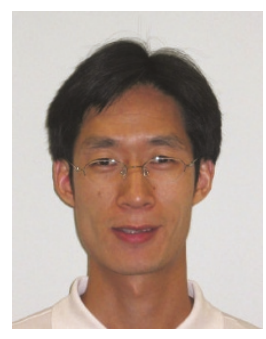

Tiecheng Liu received the M.S. degree in electrical engineering from Chinese Academy of Sciences in 1999 and Ph.D. degree in computer science from Columbia University in 2003. Since 2003, he was with the Department of Computer Science and Engineering at the University of South Carolina, where he is now an Assistant Professor. His main research interests include computer vision, image and video process-

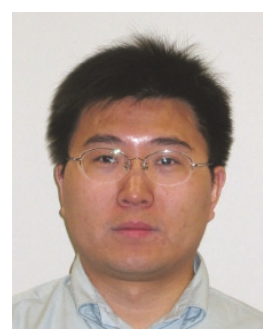
ing, and multimedia and advanced learning technologies. He published many refereed papers in the area of computer vision and multimedia technology and served as Committee Member for the IEEE MCBAR '04, CIVR '05, and other conferences. He is a Member of the IEEE, ACM, and USC chapter of UPE. 